\title{
A note on the Neyman-Rayner triangle
}

\author{
A. G. Shannon \\ Warrane College, The University of New South Wales \\ Kensington, NSW, 2033, Australia \\ e-mail: tshannon38@gmail.com
}

Received: 30 September 2021

Accepted: 2 November 2021

\begin{abstract}
This note raises questions for other number theorists to tackle. It considers a triangle arising from some statistical research of John Rayner and his use of some orthonormal polynomials related to the Legendre polynomials. These are expressed in a way that challenges the generalizing them. In particular, the coefficients are expressed in a triangle and related to known sequences in the Online Encyclopedia of Integer Sequences. The note actually raises more questions than it answers when it links with the cluster algebra of Fomin and Zelevinsky.
\end{abstract} Keywords: Neyman-Rayner triangle, Orthonormal polynomials, Legendre polynomials, Cluster algebra.

2020 Mathematics Subject Classifications: 11S05, 11C08, 11N30.

\section{Introduction}

Rayner and Best [7] point out that "the concept of smooth goodness of fitness tests was introduced in Neyman [5]" but goodness of fit concepts in general usually go back to Karl Pearson [6]. Rayner further pointed out that Jerzy Neyman's smooth alternative of order $k$ to the uniform distribution on $(0,1)$ has probability density for

$$
h_{k}(y, \theta)=\exp \left\{\sum_{i=1}^{k} \theta_{i} \pi_{i}(y)-K(\theta)\right\}, \quad 0<y<1, \quad k=1,2, \ldots
$$

where $K(\theta)$ is a normalising constant and the $\pi_{i}(y)$ are orthonormal polynomials related to the Legendre polynomials [2].

It is the purpose of this note to consider some number theoretic properties of the $\pi_{i}(y)$ polynomials ( $i=0,1,2,3,4$ in Rayner) which, for convenience, we label as Neyman-Rayner (N-R) polynomials. 


\section{N-R polynomials}

Rayner lists the first five such polynomials, slightly modified below. We also add some more in order to build up a picture of patterns.

$$
\begin{aligned}
& \pi_{0}(y)=\sqrt{1}(1) \\
& \pi_{1}(y)=\sqrt{3}(2 y-1) \\
& \pi_{2}(y)=\sqrt{5}\left(6 y^{2}-6 y+1\right) \\
& \pi_{3}(y)=\sqrt{ } 7\left(20 y^{3}-30 y^{2}+12 y-1\right) \\
& \pi_{4}(y)=\sqrt{9}\left(70 y^{4}-140 y^{3}+90 y^{2}-20 y+1\right) \\
& \pi_{5}(y)=\sqrt{11}\left(252 y^{5}-630 y^{4}+560 y^{3}-210 y^{2}+30 y-1\right) \\
& \pi_{6}(y)=\sqrt{13}\left(924 y^{6}-2772 y^{5}+3150 y^{4}-1680 y^{3}+420 y^{2}-42 y+1\right) .
\end{aligned}
$$

The first challenge is to generalize this.

\section{N-R triangle}

We assemble the absolute values of the polynomial coefficients into a triangle, as the row sums are all unity if we include the signed values of the coefficients. The row sums are in the rightmost column, and the pertinent OIES references [8] are in the bottom row. Further properties can probably be found since the triangle is essentially the same the triangle as $T(n, k)$, the number of compatible $k$-sets of cluster variables in [4] as set out in A063007 of [8], though there would seem to be number theory and related combinatorial life left in the triangle as well as in the polynomials.

\begin{tabular}{|c|r|r|r|r|r|r|r|r|}
\hline $\boldsymbol{i}$ & \multicolumn{7}{|c|}{ Signed values of coefficients } & Row sum \\
\hline 0 & 1 & & & & & & & 1 \\
\hline 1 & 2 & 1 & & & & & & 3 \\
\hline 2 & 6 & 6 & 1 & & & & & 13 \\
\hline 3 & 20 & 30 & 12 & 1 & & & & 63 \\
\hline 4 & 70 & 140 & 90 & 20 & 1 & & & 321 \\
\hline 5 & 252 & 630 & 560 & 210 & 30 & 1 & & 1683 \\
\hline 6 & 924 & 2772 & 3150 & 1680 & 420 & 42 & 1 & 8989 \\
\hline OEIS & A000984 & A002457 & A002544 & A007744 & A106440 & A013613 & A033999 & A001850 \\
\hline
\end{tabular}

The next challenge is to see if the associated polynomials can be modified in any way so that analogues of the Turán expressions for the Hermite polynomials [1] and generating functions for the Bessel functions [3] can be found. 


\section{References}

[1] Al-Salam, W. (1961). A generalized Turán expression for the Bessel functions. The American Mathematical Monthly, 68(2), 146-149.

[2] Bera, A. K., \& Ghosh, A. (2019). Neyman's Smooth Test and its Applications in Econometrics. In A. Ullah, A. T. K. Wan \& A. Chaturverdi (Ed.), Handbook of Applied Econometrics and Statistical Inference. (pp. 177-230). Baton Rouge, FL: CRC Press.

[3] Dattoli, G., Migliorati, M., \& Srivastava, H. M. (2004). Some families of generating functions for the Bessel and related functions. Georgian Mathematical Journal, 11(2), 219-228.

[4] Fomin, S. \& Zelevinsky, A. (2002). Cluster algebras I: Foundations. Journal of the American Mathematical Society, 15(2), 497-529.

[5] Neyman, J. (1937). 'Smooth' test for goodness of fit. Scandinavian Actuarial Journal, 3-4, 149-199.

[6] Plackett, R. L. (1983). Karl Pearson and the Chi-Squared Test. International Statistical Review, 51(1), 59-72.

[7] Rayner, J. W. C., \& Best, D. J. (1986). Neyman-type Smooth Tests for Location-Scale Families. Biometrika, 73(2), 437-446.

[8] Sloane, N. J. A. (1964). The On-Line Encyclopedia of Integer Sequences. Retrieved from: https://oeis.org. 